\title{
The anti-proliferative effect of TI1B, a major Bowman-Birk isoinhibitor from pea (Pisum sativum L.), on HT29 colon cancer cells is mediated through protease inhibition
}

\author{
Alfonso Clemente $^{1 *}$, M. Carmen Marín-Manzano ${ }^{1}$, Elisabeth Jiménez ${ }^{1}$, M. Carmen Arques ${ }^{1}$ \\ and Claire Domoney ${ }^{2}$ \\ ${ }^{1}$ Department of Physiology and Biochemistry of Nutrition, Estación Experimental del Zaidin (CSIC), Professor Albareda 1, \\ 18008 Granada, Spain \\ ${ }^{2}$ Department of Metabolic Biology, John Innes Centre, Norwich Research Park, Norwich NR4 7UH, UK
}

(Submitted 15 September 2011 - Accepted 24 October 2011)

\section{Abstract}

Bowman-Birk inhibitors (BBI) from legumes, such as soyabean, pea, lentil and chickpea, are naturally occurring plant protease inhibitors which have potential health-promoting properties within the mammalian gastrointestinal tract. BBI can survive both acidic conditions and the action of proteolytic enzymes within the stomach and small intestine, permitting significant amounts to reach the large intestine in active form to exert their reported anti-carcinogenic and anti-inflammatory properties. In a previous study, we reported the ability of a recombinant form of T11B (rT11B), representing a major BBI isoinhibitor from pea, to influence negatively the growth of human colorectal adenocarcinoma HT29 cells in vitro. In the present study, we investigate if this effect is related directly to the intrinsic ability of BBI to inhibit serine proteases. $\mathrm{rTI1B}$ and a novel engineered mutant, having amino acid substitutions at the $\mathrm{P}_{1}$ positions in the two inhibitory domains, were expressed in the yeast Pichia pastoris. The rTI1B proved to be active against trypsin and chymotrypsin, showing $K_{\mathrm{i}}$ values at nanomolar concentrations, whereas the related mutant protein was inactive against both serine proteases. The proliferation of HT29 colon cancer cells was significantly affected by rTI1B in a dose-dependent manner $\left(\mathrm{IC}_{50}=31\right.$ (SD 7) $\mu \mathrm{M}$ ), whereas the inactive mutant did not show any significant effect on colon cancer cell growth. In addition, neither recombinant protein affected the growth of non-malignant colonic fibroblast CCD-18Co cells. These findings suggest that serine proteases should be considered as important targets in investigating the potential chemopreventive role of BBI during the early stages of colorectal carcinogenesis.

\section{Key words: Bowman-Birk inhibitors: Cell proliferation: Colorectal cancer cells: Pea: Protease inhibition: Serine proteases}

Colorectal cancer has become one of the leading causes of cancer-related deaths worldwide and is currently increasing in Western countries ${ }^{(1)}$; consequently, much attention has been focused on preventive and therapeutic strategies. The beneficial effects of certain bioactive compounds, mainly present in fruits and vegetables, in preventing and treating colorectal cancer have become a major focus of research ${ }^{(2-4)}$. Among these, Bowman-Birk inhibitors (BBI) from legumes, such as soyabean (Glycine max), pea (Pisum sativum), lentil (Lens culinaris) and chickpea (Cicer arietinum), have been shown to have potential in protecting against inflammatory disorders and cancer development within the mammalian gastrointestinal tract $^{(5-8)}$. BBI are naturally occurring protease inhibitors with the ability to inhibit specifically serine (predominantly trypsin- and chymotrypsin-like) proteases. BBI are extensively disulphide-linked within proteins and have been demonstrated to be structurally and functionally resistant to the challenges (acidic conditions and the action of proteolytic enzymes) of the gastrointestinal tract in vivo. The conformational rigidity of $\mathrm{BBI}$ linked to the number and distribution of intramolecular disulphide bonds is mostly responsible for the high stability of these proteins towards extreme conditions and helps to maintain the structural and functional features of their binding loops ${ }^{(9-11)}$. BBI transit through the stomach and small intestine without major degradation, permitting significant amounts $(5-8 \%$ of

\footnotetext{
Abbreviations: BAPNA, $N$ - $\alpha$-benzoyl-DL-arginine-p-nitroanilide; BBI, Bowman-Birk inhibitors; BTEE, $N$-benzoyl-L-tyrosine ethyl ester; cDNA, complementary DNA; CIA, chymotrypsin inhibitor activity; DMEM, Dulbecco's modified Eagle's medium; IC ${ }_{50}$, inhibitory concentration showing $50 \%$ effect; NR, neutral red; rTI1B, recombinant TI1B; TIA, trypsin inhibitor activity.
} 
total ingested) to reach the large intestine in active form ${ }^{(12)}$. In addition, the biological activity of BBI is largely unaffected by the enzymatic and metabolic activity of faecal microbiota, retaining significance as a bioactive compound in the large intestine ${ }^{(13)}$.

In vitro and in vivo studies suggest that soyabean BBI can exert a protective and/or suppressive effect on cancer development within the gastrointestinal tract. Purified soyabean BBI and BBI concentrate, a protein extract of soyabean enriched in $\mathrm{BBI}^{(14)}$, were both effective at concentrations as low as $10 \mathrm{mg} / 100 \mathrm{~g}$ of diet in reducing the incidence and frequency of colorectal tumours in the dimethylhydrazine rat model, without any adverse side effects documented for animal growth or organ physiology ${ }^{(5)}$. Autoclaved BBI concentrate, in which the serine protease inhibitory activity of BBI was abolished, did not show any significant suppressive effect on colon tumour development, suggesting that the intrinsic ability of soyabean BBI to inhibit serine proteases may be required for their colorectal chemopreventive properties. Human epithelial cell lines derived from colon carcinoma are well-established models to investigate in vitro the action mechanism by which dietary compounds function in a chemoprotective role during the early stages of carcinogenesis. Recent studies have demonstrated a significant concentrationand time-dependent decrease in the growth of HT29 human colon adenocarcinoma cells in vitro, following treatment with soyabean $\mathrm{BBI}^{(15)}$. Similar effects have been reported for BBI from other legume sources, including those from pea ${ }^{(16)}$ and lentil ${ }^{(17)}$; the $\mathrm{IC}_{50}$ values (inhibitory concentration showing $50 \%$ effect) for these protease inhibitors ranged from 32 to $73 \mu \mathrm{m}$. Interestingly, soyabean BBI that had been chemically inactivated did not demonstrate any significant effect on the proliferation of colon cancer cells, suggesting that a native conformation is necessary for BBI to exert anti-proliferative effects on such cells ${ }^{(15)}$.

In pea, the TI1 gene class encodes BBI that are expressed predominantly within seeds. TI1 is synthesised as a precursor protein of 114 amino acids, with a deduced mature peptide region of seventy-two amino acids having the ability to inhibit potently trypsin- and chymotrypsin-like proteases at the first and second inhibitory domains, respectively ${ }^{(18,19)}$. In a previous study, we reported the inhibition of the growth in vitro of HT29 colon adenocarcinoma cells by a recombinant form of TI1 from the pea $\mathrm{cv}$. Birte $(\mathrm{rTI} 1 \mathrm{~B})^{(16)}$. In the present study, we have evaluated the functional properties and antiproliferative effects of rTI1B, expressed heterologously in Pichia pastoris, on colon cancer cells. We have compared the effects of rTI1B with those observed using a related mutant protein, obtained by site-directed mutagenesis of the $\mathrm{P}_{1}$ amino acid position in both inhibitory domains of TI1B. These novel data demonstrate the unambiguous relationship between the intrinsic ability of BBI to inhibit serine proteases and the associated negative effects on colon cancer cell growth, suggesting that serine proteases involved in early stages of carcinogenesis should be considered important targets in investigating the potential of BBI and related proteins as colorectal chemopreventive agents.

\section{Experimental methods}

\section{Materials}

Trypsin (type III) and $\alpha$-chymotrypsin (type VII) from bovine pancreas, $N$ - $\alpha$-benzoyl-DL-arginine-p-nitroanilide (BAPNA), $N$ benzoyl-L-tyrosine ethyl ester (BTEE), high-glucose Dulbecco's modified Eagle's medium (DMEM), neutral red (NR) and additional high-grade chemicals for cell culture were obtained from Sigma. The P. pastoris host strain GS115 and the pPIC9 vector for expression and secretion of recombinant BBI were obtained from Invitrogen. The human colorectal adenocarcinoma HT29 and the normal colon fibroblastic CCD-18Co cell lines were supplied by the Cell Bank of the Scientific Instrumentation Centre at the University of Granada (CIC-UGR). Culture flasks and flat bottom ninety-six-well microtitre plates were purchased from Corning Costar and Nunc, respectively. All other chemicals were of analytical grade.

\section{Growth media composition for Pichia pastoris}

The P. pastoris GS115 (Invitrogen) host strain was maintained in yeast extract peptone dextrose broth ( $10 \mathrm{~g}$ yeast extract, $20 \mathrm{~g}$ peptone, $20 \mathrm{~g}$ dextrose per litre). Minimal dextrose plates $(13.4 \mathrm{~g}$ yeast $\mathrm{N}$ base, $10 \mathrm{~g}$ glucose, $0.4 \mathrm{mg}$ biotin and $15 \mathrm{~g}$ agar per litre) were used for the selection of putative transformants. Buffered minimal glycerol broth $(13.4 \mathrm{~g}$ yeast $\mathrm{N}$ base, $0.4 \mathrm{mg}$ biotin, $100 \mathrm{ml}$ of $1 \mathrm{M}-\mathrm{K}_{2} \mathrm{HPO}_{4} / \mathrm{KH}_{2} \mathrm{PO}_{4} \mathrm{pH} \quad 6.0$ and $1 \%$ glycerol) was used until cultures reached log-phase growth. Buffered minimal methanol broth (identical to buffered minimal glycerol except for $0.5 \%$ methanol instead of $1 \%$ glycerol) was used to induce expression of the recombinant proteins.

\section{Cloning, mutagenesis and expression of TI1B and derivative in the methylotrophic yeast Pichia pastoris}

A sequence encoding a mature form of TI1B, a major BBI identified in pea seeds in vivo, was amplified from a cloned complementary DNA (cDNA) (pTI12-36) ${ }^{(19)}$, using specific primers (N_peaTI1B and C_peaTI1B) (Table 1). The $\mathrm{N}$ and C primers incorporated $X h o I$ and EcoRI cleavage sites, respectively, to allow directional cloning of the amplified DNA into the pPIC9 expression vector, used for in-frame integration of the $T I 1$ gene into the $P$. pastoris genome. The N_peaTI1B primer was designed to encode a Kex2 processing site (Lys-Arg) followed by two Glu-Ala repeats, just before the $\mathrm{N}$-terminus of TI1B, in order to increase the efficiency of Kex2 cleavage; an endogenous STE13 dipeptidyl aminopeptidase is predicted to remove both Glu-Ala dipeptides to yield the final recombinant proteins. PCR conditions were an initial denaturation step at $94^{\circ} \mathrm{C}$ for $5 \mathrm{~min}$, followed by thirty-five cycles of $94^{\circ} \mathrm{C}$ for $30 \mathrm{~s}, 56^{\circ} \mathrm{C}$ for $30 \mathrm{~s}$ and $68^{\circ} \mathrm{C}$ for $1 \mathrm{~min}$, and a final elongation step at $68^{\circ} \mathrm{C}$ for $5 \mathrm{~min}$. The amplicon obtained was digested with both XhoI and EcoRI enzymes, ligated into pPIC9 vector previously digested with the same enzymes and used to transform Escherichia coli $\mathrm{DH} 5 \alpha$ competent cells.

In order to obtain an inactive TI1B-like protein, mutations were introduced by PCR into the active-site domains, using 
Table 1. List of primers used ${ }^{\star}$

\begin{tabular}{ll}
\hline Primer name & Sequence 5'-3' \\
\hline N_peaTI1B & GTATCTCTCGAGAAAAGAGAGGCTGAAGCTGGTGATGATGTCAAATC \\
C_peaTI1B & CCTAGGGAATTCTCAGTTCTTAATGACCTCCT \\
F1 & GCCTTTGCACAGGATCAAACCCTCC \\
R1 & GGAGGGTTTGATCCTGTGCAAAGGC \\
F2 & GCATTTGTGCAGGTTCTAATCCTCC \\
R2 & GGAGGATTAGAACC TGCACAAATGC \\
$5^{\prime}$ AOX1 & GACTGGTTCCAATTGACAAGC \\
3 $^{\prime}$ AOX1 & GCAAATGGCATTCTGACATCC \\
\hline \\
*The Xhol and ECoRI cleavage sites are underlined, the Kex2 processing site encoding Lys-Arg is in bold text, \\
the Glu-Ala repeats are italicised and the mutations are in grey boxes. 5'AOX1 and 3'AOX1 are the sequen- $^{\prime}$ cing primers.
\end{tabular}

the overlap expression method ${ }^{(20)}$. The cDNA encoding TI1B was used as a template; the specific mutagenic primer pairs (F1-R1 and F2-R2, see Table1) were designed to introduce the amino acid Gly instead of $\mathrm{Lys}^{16}$ and $\mathrm{Tyr}^{42}$ at the $\mathrm{P}_{1}$ positions of the trypsin and chymotrypsin inhibitory domains, respectively. The N_peaTI1B and C_peaTI1B primers were used to amplify the final PCR amplified product, which was digested with XhoI and Eco RI, ligated into pPIC9 vector and used to transform $\mathrm{DH} 5 \alpha$ cells as described previously. All PCR experiments were performed using a mixture of Platinum Pfx (Invitrogen) and MBL-Taq DNA polymerase (Dominion-MBL)

Plasmids containing inserts were selected with ampicillin $(100 \mu \mathrm{g} / \mathrm{ml}$ medium) and the sequences verified by using the $5^{\prime} \mathrm{AOX} 1$ and $3^{\prime} \mathrm{AOX} 1$ primers (Table 1) on the pPIC9 vector. For efficient integration into the his 4 locus of the $P$. pastoris genome, pPIC9-derived plasmids were first linearised by overnight digestion with $\mathrm{Sal} \mathrm{I}$ at $37^{\circ} \mathrm{C}$, and digested DNA $(5 \mu \mathrm{g})$ used to transform $P$. pastoris GS115 strain by electroporation (Gene Pulser XCell, Bio-Rad Laboratories). As a control, expression vector without any insert was used to transform $P$. pastoris. Transformants were selected on minimal dextrose agar plates without histidine, according to the Pichia expression kit manual (Invitrogen, a manual of methods for expression of recombinant proteins in P. pastoris, K1710-01). Up to sixteen individual colonies containing $T I 1 B$ or its derivative mutant were grown overnight at $30^{\circ} \mathrm{C}$ in $100 \mathrm{ml}$ of buffered minimal glycerol; cells were pelleted and resuspended in $20 \mathrm{ml}$ of buffered minimal methanol. Incubation was carried out in an orbital shaker $\left(250 \mathrm{rpm}, 28^{\circ} \mathrm{C}\right)$ for $6 \mathrm{~d}$, with methanol being added $(0.5 \%, \mathrm{v} / \mathrm{v})$ every $24 \mathrm{~h}$ to compensate for consumption and evaporation. Strains secreting the highest levels of rTI1B or the corresponding mutant protein were selected for large-scale protein expression; selection was based on SDS-PAGE analysis and, where appropriate, trypsin inhibitor activity (TIA) and chymotrypsin inhibitor activity (CIA) assays (see the following text)

\section{Protein expression and purification}

The selected yeast transformants were inoculated in $40 \mathrm{ml}$ of yeast extract peptone dextrose medium, cultured overnight at $28^{\circ} \mathrm{C}$ and then used to inoculate 4 litres of buffered minimal glycerol. When an $\mathrm{OD}_{600 \mathrm{~nm}}$ in the range 2-6 was reached, cells were pelleted and resuspended in $800 \mathrm{ml}$ of buffered minimal methanol medium. Cultures were then grown for $6 \mathrm{~d}$ at $28^{\circ} \mathrm{C}$, and supplemented daily with $0.5 \%(\mathrm{v} / \mathrm{v})$ methanol to maintain the induced protein expression. Cells were pelleted and the supernatants (spent media) dialysed extensively against distilled water and freeze-dried. Protein extracts from freeze-dried dialysates were fractionated on a MonoS 5/50 GL cation exchange column (GE Healthcare), connected to an AKTA FPLC system (GE Healthcare), using a linear gradient of $0-0.22 \mathrm{M}-\mathrm{NaCl}$ in $50 \mathrm{~mm}$-sodium acetate buffer, $\mathrm{pH} 4 \cdot 4$, at a flow rate of $1 \mathrm{ml} / \mathrm{min}$. The elution was monitored at $280 \mathrm{~nm}$ and $0.5 \mathrm{ml}$ fractions were collected. TIA measurements of eluted samples were carried out in flat-bottom microtitre plates and assay products measured at $\mathrm{OD}_{405 \mathrm{~nm}}$ as previously described ${ }^{(12)}$. CIA evaluation of eluted samples was carried out as described below. For rTI1B, column fractions containing inhibitory activity were pooled, dialysed extensively against distilled water and freeze-dried until use. In the case of the mutant form of rTI1B, where TIA and CIA were expected to be low or completely abolished, monitoring of the elution profile was carried out by SDS-PAGE.

\section{Peptide mass fingerprinting and molecular mass determination of recombinant proteins}

The recombinant proteins $(10 \mu \mathrm{g})$ were dissolved in NuPAGE lithium dodecyl sulphate sample buffer (Invitrogen) and separated by electrophoresis on Novex $12 \%$ Bis-Tris pre-cast gels using 2-N-morpholine-ethane sulphonic acid (NuPAGE MES, Invitrogen) as running buffer. Immediately before use, samples were reduced with dithiothreitol (DTT) and NuPAGE antioxidant added to the upper buffer chamber to prevent re-oxidation of reduced proteins during electrophoresis. Bands were excised from Colloidal Blue (Invitrogen)stained gels and subjected to in-gel trypsin digestion. Peptide fragments from digested proteins were desalted and concentrated using C-18 ZipTip columns (Millipore) and then loaded directly onto the matrix-assisted laser desorption/ ionization (MALDI) plate, using $\alpha$-cyano-4-hydroxycinnamic acid as the matrix for MALDI-MS analysis. MS spectra were obtained automatically in a 4700 Proteomics Analyzer (Applied Biosystems) operating in reflectron mode with delayed extraction. Peptide mass data were used for protein identification against the MS protein sequence database 
(www.matrixscience.com). Additionally, a further attempt to identify the peptide mass data against the theoretical mass peptides derived from trypsin digestion of the rTI1B mutant, not included in databases, was carried out.

Freeze-dried purified proteins were dissolved in $1 \%(\mathrm{v} / \mathrm{v})$ trifluoroacetic acid (TFA), mixed with an equal volume of matrix $(3 \mathrm{mg} / \mathrm{ml}$ of $\alpha$-cyano-4-hydroxycinnamic acid in $70 \%$ (v/v) acetonitrile/ $0 \cdot 1 \%$ TFA), deposited onto a MALDI sample probe and dried under ambient conditions. MS spectra were obtained automatically in a 4700 Proteomics Analyzer (Applied Biosystems), in the $800-10000 \mathrm{~m} / z$ range, and average molecular masses of recombinant proteins were determined.

\section{Measurement of protease inhibitory activities}

rTI1B and its mutant derivative were assessed for TIA and CIA. TIA was measured using a modified small-scale quantitative assay with BAPNA as specific substrate, and using $50 \mathrm{~mm}$ Tris, pH 7.5 as enzyme assay buffer. One trypsin inhibitor unit was defined as that which gives a reduction in absorbance at $410 \mathrm{~nm}$ of 0.01 , relative to trypsin control reactions, in $10 \mathrm{~min}$ in a defined assay volume of $10 \mathrm{ml}^{(21)}$. CIA was measured using BTEE as specific substrate. One chymotrypsin inhibitor unit was defined as that which gives a reduction in absorbance at $256 \mathrm{~nm}$ of $0 \cdot 01$, relative to chymotrypsin control reactions, in $5 \mathrm{~min}$ in a defined assay volume of $10 \mathrm{ml}$, as described previously ${ }^{(22)}$. Specific TIA and CIA of the recombinant proteins, expressed as inhibitor units per mg of protein, were calculated. The inhibition constants $\left(K_{\mathrm{i}}\right)$ of rTI1B for trypsin ( $\mathrm{pH} 7 \cdot 5)$ and chymotrypsin $(\mathrm{pH} 7 \cdot 8)$, were determined from dose-response curves by competitive assays, using the chromogenic substrates BAPNA and BTEE, respectively ${ }^{(22)}$. The reactions were initiated by adding trypsin ( $108 \mathrm{~nm}$ ) or chymotrypsin $(28 \mathrm{~nm})$ with the respective substrate concentrations determined by $K_{\mathrm{m}}$ measurements. The concentration of rTI1B required to achieve a half-maximal degree of inhibition $\left(\mathrm{IC}_{50}\right)$ was determined for each protease, using the GraFit software (GraFit version 5, Erithacus Software Limited). $K_{\mathrm{i}}$ were calculated from $\mathrm{IC}_{50}$ values using the tight-binding equations for competitive inhibitors ${ }^{(23)}$. The trypsin and chymotrypsin inhibitory patterns of rTI1B and its mutant derivative were analysed on $4-16 \%$ zymogram blue casein gels (Invitrogen), in comparisons with the native proteins present in an albumin fraction from pea seeds ${ }^{(22)}$. After electrophoresis, and following the manufacturer's instructions, gels were treated with zymogram renaturating buffer (Invitrogen) for $30 \mathrm{~min}$ at room temperature, equilibrated with zymogram developing buffer (Invitrogen), incubated with $25 \mathrm{ml}$ of trypsin or chymotrypsin solution $(0.08 \mathrm{mg} / \mathrm{ml}$ of zymogram developing buffer) at $37^{\circ} \mathrm{C}$ for $1.5 \mathrm{~h}$, and washed with distilled water before the addition of acetic acid to stop the enzymatic reaction.

\section{Cell viability assays}

Human colorectal adenocarcinoma HT29 and normal colon fibroblastic CCD-18Co cells were maintained by serial passage in $75 \mathrm{~cm}^{2}$ plastic culture flasks. HT29 cells were cultured in
DMEM, supplemented with fetal bovine serum (10\%), 2 mMglutamine and $1 \%$ antibiotic-antimicotic solution (Sigma, A5955), all at final concentration. In the case of CCD-18Co fibroblastic cells, media were supplemented additionally with non-essential amino acids to $1 \%$ (final w/v; Sigma, M7145). Optimal assay conditions for rapidly dividing unpolarised, undifferentiated colonic cells were reported previously $^{(16)}$. The NR (3-amino-7-dimethylamino-2-methylphenazine hydrochloride) assay was validated by performing calibration assays, where HT29 and CCD18-Co cells were seeded in ninety-six-well microtitre plates at several cell densities. Accordingly, ninety-six-well microtitre plates were inoculated at a density of 2000 HT29 cells per well in $200 \mu \mathrm{l}$ of growth media; in the case of CCD-18Co cells, 5000 cells per well were added. Plates were incubated under $5 \% \mathrm{CO}_{2}$ in humidified air for $24 \mathrm{~h}$ to allow the cells to adhere to the wells. rTI1B and its mutant derivative were dissolved in growth media at a range of concentrations $(0-61 \mu \mathrm{m})$ and added to the cells under sterile conditions. Control cells received neither form of rTI1B. At the end of the growth period $(96 \mathrm{~h})$, the viability of HT29 and CCD-Co18 cells was assessed by the NR cytotoxicity assay, based on the ability of viable uninjured cells to incorporate and actively bind NR, a supravital dye, into lysosomes. Cells were stained with NR solution $\left(2 \mathrm{~h}\right.$ at $\left.37^{\circ} \mathrm{C}\right)$, followed by cell fixation ( $0.5 \%$ formaldehyde, $0.1 \% \mathrm{CaCl}_{2}$ for $30 \mathrm{~s}$ ) at room temperature. Plates were washed by two brief immersions in PBS (0.01 M-sodium phosphate buffer, $0 \cdot 15 \mathrm{M}-\mathrm{NaCl})$ and the dye extracted from the viable cells using an acidified ethanol solution $\left(50 \%\right.$ ethanol, $1 \%$ acetic acid overnight at $\left.4^{\circ} \mathrm{C}\right)$. The absorbance of the solubilised dye was quantified at $\mathrm{OD}_{550 \mathrm{~nm}}$ using a Bio-Rad Model 550 microplate reader (Bio-Rad). Cell viability data, expressed as a percentage of the values determined for control cells grown in the absence of either form of rTI1B, were obtained from at least three independent experiments ( $n \geq 4$ per experiment). The concentration of rTI1B that reduced cell viability by $50 \%\left(\mathrm{IC}_{50}\right)$, as compared with untreated controls, was calculated by nonlinear regression fit using the GraFit software. The data were analysed statistically by the Bonferroni's test to compare means and statistical significance was set at $P<0 \cdot 05$.

\section{Results}

\section{Heterologous expression of TI1B and an engineered} mutant

A cDNA clone (pTI12-36) was chosen for heterologous expression of TI1B, a major isoinhibitor from pea seeds, in $P$. pastoris. The in silico translation of the cDNA showed two inhibitory domains and fourteen cysteine residues in conserved positions, as described previously ${ }^{(19,24)}$. Following the nomenclature of Schechter \& Berger ${ }^{(25)}$, the residues Lys and Tyr at position $\mathrm{P}_{1}$ in the first (CTKSNPPTC) and second inhibitory domain (CAYSNPPKC), respectively, confer specificity for inhibition of trypsin and chymotrypsin-like proteases. To obtain an inactive TI1B-like protein, pTI12-36 cDNA was used as a template and mutations to its active sites achieved 
by site-directed mutagenesis. Overlapping PCR was used to substitute the $P_{1}$ amino acid within the trypsin (Lys ${ }^{16}$ ) and chymotrypsin $\left(\mathrm{Tyr}^{42}\right)$ inhibitory domains with a Gly at both positions.

Pichia pastoris was selected for the expression of rTI1B and its mutant derivative since this host had been shown previously to be capable of folding and secreting a BBI from lentil ${ }^{(17)}$. Sequences corresponding to the pea CDNA, pTI1236, encoding either a mature form of TI1B lacking any of the forty-two amino acid prepropeptide sequence, or the mutant derivative of TI1B predicted to be inactive, were cloned into the vector pPIC9 downstream of the $\alpha$-factor secretion signal sequence to direct the recombinant proteins into the secretory pathway of $P$. pastoris. Expression of both recombinant forms of TI1B was induced by methanol, and the recombinant proteins were subsequently secreted into the culture supernatants. TIA and CIA assays were used for rapid screening of a large number of putative TI1B transformants after induction with methanol; spent media from transformants expressing the mutant form of rTIB lacked inhibitory activity in these assays (not shown). In the culture supernatants of putative transformants of rTI1B and its mutant derivative, polypeptides in the range $7-9 \mathrm{kDa}$ were detected by SDS-PAGE; in the case of yeast transformed with the empty vector, these polypeptides were absent from culture supernatants (not shown). Yeast transformants expressing the highest levels of rTI1B were selected by TIA and CIA for subsequent large-scale protein expression, whereas transformants secreting the rTI1B mutant were selected by SDS-PAGE followed by protein concentration determination, as previously described $^{(22)}$. Zymography of culture supernatants of rTI1B under non-denaturing conditions allowed the detection of two bands with specific inhibitory activities against the digestive enzymes trypsin and chymotrypsin (Fig. 1). These bands showed an increased mobility on non-denaturing gels when compared with the primary mature product of the TI1 gene observed in vivo. In zymograms, the overall charges of the rTI1B forms were observed to be -3 and -4 , whereas that deduced from the sequence of the mature TI1B variant in vivo is $-2^{(18)}$. Culture supernatants from the rTI1B mutant derivative failed to inhibit in-gel activity of either digestive enzyme (Fig. 1).

\section{Purification, molecular and functional characterisation of the recombinant proteins}

To purify the recombinant proteins, secreted proteins were fractionated by Monos cation exchange chromatography. The elution patterns of rTI1B and its inactive mutant are shown in Fig. 2(A) and (B), respectively. The recombinant proteins were retained by the Mono $\mathrm{S}$ column at $\mathrm{pH} 4.4$. In good agreement with results obtained from non-denaturing gels, rTI1B was resolved as two chromatographic peaks, which eluted in the range $0 \cdot 07-0 \cdot 10 \mathrm{M}-\mathrm{NaCl}$ (peak 1) and $0 \cdot 13-0 \cdot 16 \mathrm{M}-\mathrm{NaCl}$ (peak 2), and the two chromatographic peaks showed both TIA and CIA. The rTI1B mutant derivative (see later for positive identification) was eluted as a single broad chromatographic peak in the range $0 \cdot 04-0 \cdot 09 \mathrm{M}-\mathrm{NaCl}$,
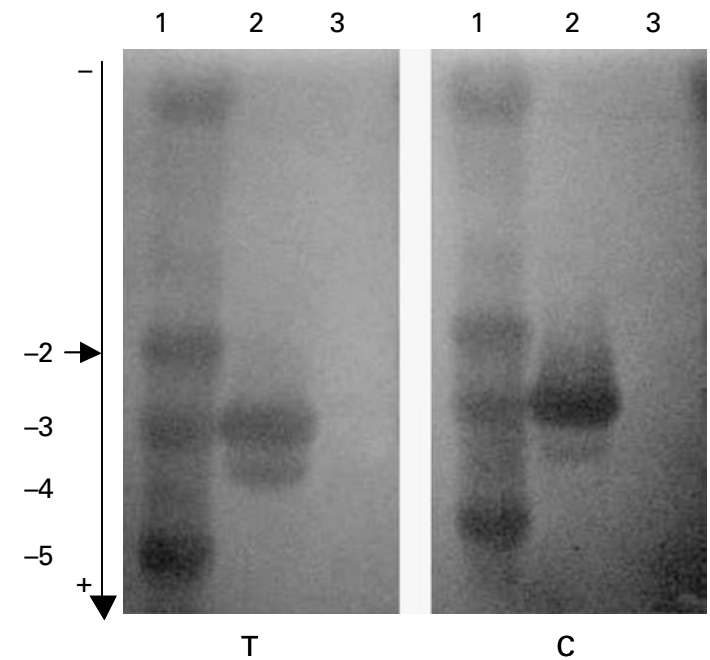

Fig. 1. In-gel protease inhibitory activity analyses of recombinant TI1B (rTI1B) and its inactive mutant. Zymogram blue casein gels were treated with the digestive enzymes, trypsin (T) or chymotrypsin (C); dark areas indicate where the enzyme has been inhibited. Lane 1: pea seed albumin fraction from cv. Birte; lane 2: culture supernatant from secreted expression of rTI1B lane 3: culture supernatant from secreted expression of the mutant rTI1B predicted to be inactive. Lane 1 contained $1.5 \mathrm{mg}$ of pea albumin fraction, whereas lanes 2 and 3 contained $20 \mu \mathrm{g}$ of recombinant protein. The direction of electrophoresis on non-denaturing gels is indicated (vertical arrow), alongside the overall charge of the major isoinhibitors found in pea seeds of $\mathrm{cv}$. Birte. Among the isoforms present in seeds, the -2 variant represents the primary mature product of the TI1B gene (horizontal arrow).

having neither TIA nor CIA (Fig. 2(B)). Differences in the elution pattern of the two recombinant proteins can be predicted to be due to the substitution of $\operatorname{Lys}^{16}$ at the $\mathrm{P}_{1}$ position of the trypsin inhibitory domain with Gly in the rTI1B mutant. The chromatographic fractions containing the recombinant proteins were pooled and analysed by SDS-PAGE; in all cases, purified fractions showed proteins in the range 7-9kDa. The peak 2 from rTI1B showed a reduced gel migration when compared to peak 1 , with the latter possibly representing a processed variant (Fig. 2(C)). Further studies by MALDI-time of flight (TOF) MS and mass peptide fingerprinting were carried out in order to determine the authenticity of the recombinant proteins. The experimentally determined $\mathrm{m} / \mathrm{z}$ values of peaks 1 and 2 derived from rTI1B were 7694 and $8146 \mathrm{Da}$, respectively, clearly differing from the value predicted for mature TI1B, allowing for disulphide formation (7946 Da) (Fig. 3). In the case of the inactive mutant, a mixture of three differentially processed isoinhibitors was deduced, based on molecular masses of 7386, 7515 and $7968 \mathrm{Da}$, compared with an expected mass of $7768 \mathrm{Da}$ for the mutant construct with correct disulphide bond formation. The presence of these three forms was not revealed by SDS-PAGE; this may be due to differences in their relative proportions. Given their lack of inhibitory activity, further chromatographic separation of inactive forms was not carried out before cell assays. In addition, the amino acid sequences of the two recombinant proteins were matched by mass peptide fingerprinting (Fig. 3). In-gel tryptic digestion of excised electrophoretic bands was performed followed by separation 

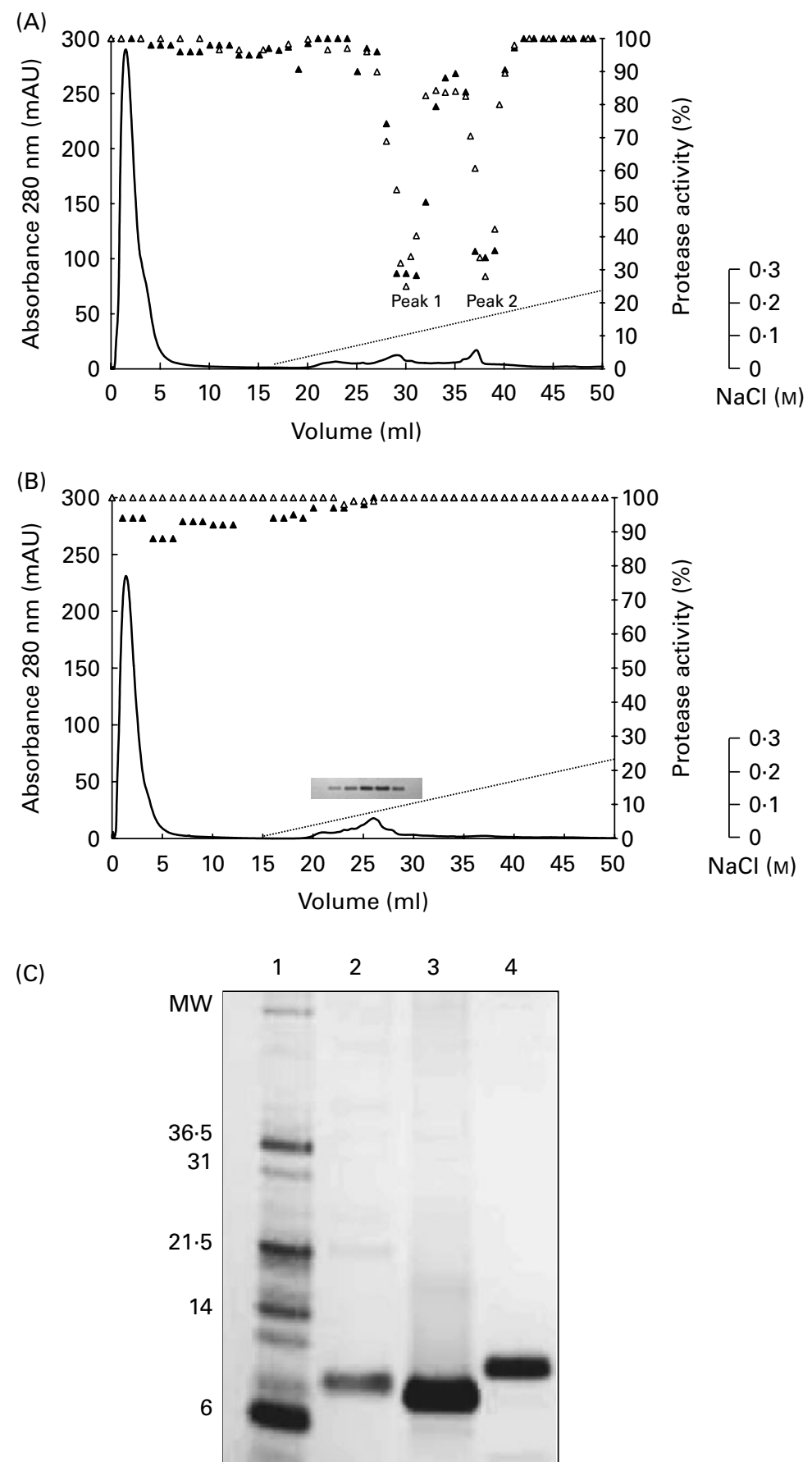

Fig. 2. Elution profile of (A) recombinant TI1B ( $r T 11 B$ ) and (B) its mutant derivative on a MonoS $5 / 50 \mathrm{GL}$ cation exchange column. Absorbance (mAU) at $280 \mathrm{~nm}$ of the chromatographic elution and the linear gradient of $\mathrm{NaCl}(0-0.22 \mathrm{M})$ are shown (solid and dotted lines, respectively). Using $\mathrm{N}$ - $\alpha$-benzoyl-DL-arginine-p-nitroanilide and $N$-benzoyl-L-tyrosine ethyl ester as specific substrates, the trypsin $(\mathbf{\Lambda})$ and chymotrypsin $(\Delta)$ inhibitory activities, measured on every fraction are shown. Where there was little or no inhibition (B), then monitoring of the elution profile was carried out by SDS-PAGE (gel inset in B); (C) SDS-PAGE under denaturing and reducing conditions of the inactive mutant protein (lane 2) and peaks 1 and 2 from rTI1B (lanes 3 and 4, respectively), following the chromatography steps. Molecular weight (MW) markers are shown in lane 1.

of the peptides generated and MS-based analysis. A search of peptide mass data against the MS protein sequence database enabled the unambiguous identification of TI1B (Swiss-Prot entry: IBBA_PEA). The sequence coverage of peaks 1 and 2 from rTI1B was 86 and $75 \%$, respectively. An attempt to identify the peptide mass data against the theoretical mass peptides derived from trypsin digestion of the rTI1B mutant, not included in databases, was also carried out. Sequence coverage of the inactive mutant was $82 \%$ and included both inhibitory domains having their corresponding Gly substitution. Although endoproteolytic removal of the signal sequence by the $P$. pastoris Kex2 protease clearly occurred, differences 


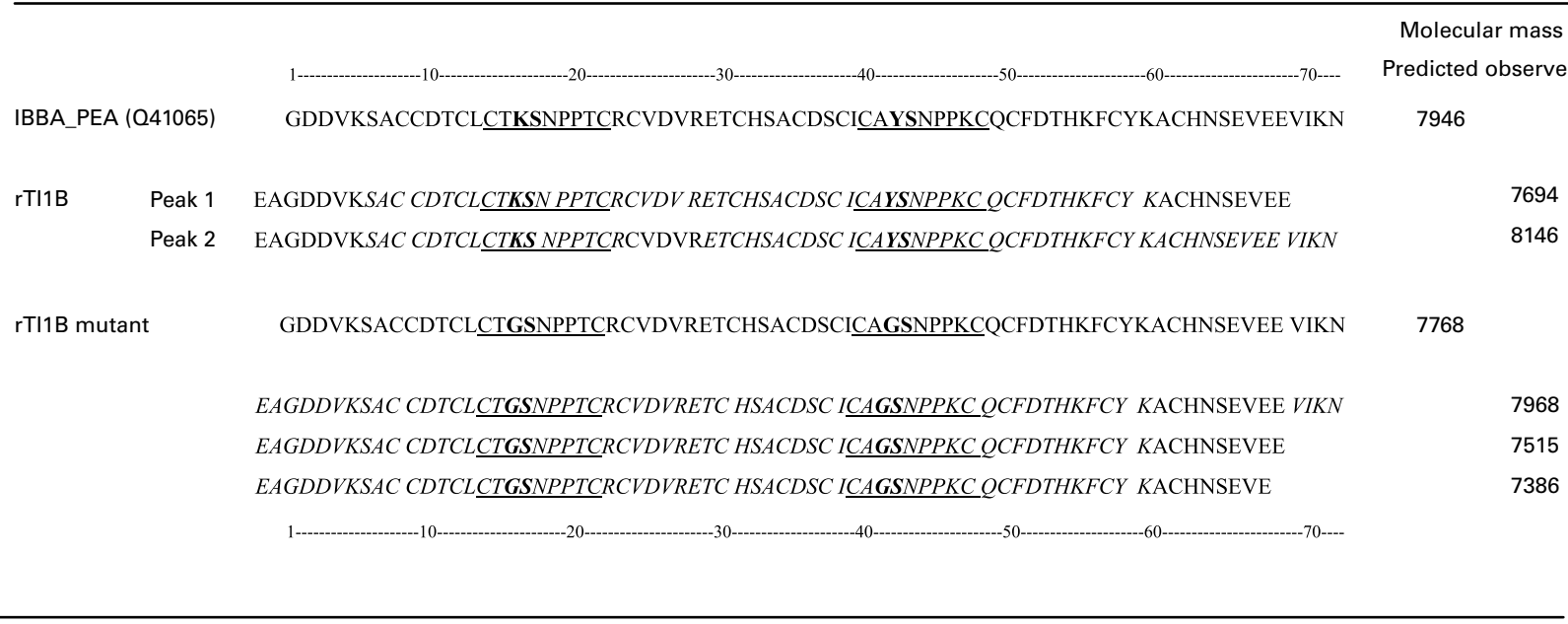

Fig. 3. Molecular mass determination and amino acid sequences deduced by peptide mass fingerprinting of recombinant Tl1B ( $\mathrm{rTl1B}$ ) and its mutant derivative, compared with the primary mature pea protein encoded by the TI1B gene (Q41065, Swiss-Prot database, Swiss Institute of Bioinformatics). Amino acid sequences of inhibitory domains are underlined. $\mathrm{P}_{1}-\mathrm{P}_{1}{ }^{\prime}$ are the reactive peptide bond sites, in bold text. $L y s(K)$ and Tyr $(\mathrm{Y})$ at position $\mathrm{P}_{1}$ determine specificity for trypsin and chymotrypsin, respectively. Amino acid sequences of recombinant proteins were deduced from mass peptide fingerprinting analyses; the peptide sequences determined for recombinant proteins are italicised. The molecular masses of the recombinant variants, determined by MALDI-TOF, are shown compared with their predicted values. The sequence Glu Ala Glu Ala was engineered just before the N-terminus of recombinant proteins for efficient cleavage by Pichia Kex2. The deduced sequences of the recombinant proteins indicate an inefficient cleavage of the dipeptide Glu Ala by the endogenous STE13 dipeptidyl aminopeptidase.

in mobility on non-denaturing gels (Fig. 1), molecular mass determination and mass peptide fingerprinting data (Fig. 3) supported the existence of a Glu-Ala extension (200 Da) at the N-terminus of the recombinant proteins (Fig. 3); this indicates an inefficiency in cleavage by the endogenous STE13 dipeptidyl aminopeptidase ${ }^{(26,27)}$. Recombinant forms having the complete Glu-Ala-Glu-Ala extension or those fully processed by STE13 were not observed. In the case of peak 1 from rTI1B, a loss of four amino acids (Val Ile Lys Asn) at the C-terminus was deduced whereas, in the case of the inactive mutant, a loss of an additional amino acid (Glu Val Ile Lys Asn) was deduced for one isoform, compared with those predicted from the DNA sequences (Fig. 3). Both an N-terminal extension (Glu Ala) and deduced processing at the C-terminus, involving loss of charged amino acid(s), are in good agreement with the observed mobility on nondenaturing gels of rTI1B (peaks 1 and 2) when compared with the primary mature product of the TI1 gene observed in vivo (Fig. 1). The mass and peptide data obtained for the mutant protein are consistent with correct protein folding, based on disulphide bond formation, and the $m / z$ variants are consistent with differential processing of C-terminal sequences, as indicated in Fig. 3. All further experiments, including kinetic and cells assays, were performed with unprocessed rTI1B (peak 2) and the mixture of isoinhibitors derived from the inactive mutant (Fig. 3).

The ability of rTI1B to inhibit the digestive enzymes trypsin and chymotrypsin was evaluated (Table 2). The specific TIA and CIA of this recombinant protein were 2476 (SD 238) and 2956 (SD 196) units per $\mathrm{mg}$ of protein, respectively. The rTI1B mutant was unable to inhibit either enzyme. Based on $\mathrm{IC}_{50}$ and $K_{\mathrm{i}}$ calculations, rTI1B was demonstrated to be a potent inhibitor of trypsin ( $K_{\mathrm{i}}$ of 21 (SD 2) nM) and chymotrypsin ( $K_{\mathrm{i}}$ of 8 (SD 1$) \mathrm{nm}$ ) (Table 2 ). Such values fall within the nanomolar range reported previously for various members of the BBI family, including those from soyabean ${ }^{(15)}$, lentil ${ }^{(17)}$ and lupin (Lupinus albus) ${ }^{(28)}$, and suggested that TI1B was expressed as a correctly folded active recombinant protein from $P$. pastoris.

Table 2. Inhibition constant $\left(K_{\mathrm{i}}\right)$ and specific inhibitory activity (IU) for trypsin (T) and chymotrysin (C) of recombinant TI1B (rTI1B) and its mutant derivative*

(Mean values and standard deviations from at least five independent determinations)

\begin{tabular}{|c|c|c|c|c|c|c|c|c|c|c|}
\hline \multirow[b]{3}{*}{ Recombinant protein } & \multirow{2}{*}{\multicolumn{2}{|c|}{$\begin{array}{l}\text { Amino acid sequence of } \\
\text { inhibitory domains }\end{array}$}} & \multicolumn{4}{|c|}{$K_{\mathrm{i}}(\mathrm{nM})$} & \multicolumn{4}{|c|}{$\begin{array}{l}\text { Specific inhibitory activity } \\
\text { (IU/mg protein) }\end{array}$} \\
\hline & & & \multicolumn{2}{|c|}{$\mathrm{T}$} & \multicolumn{2}{|c|}{ C } & \multicolumn{2}{|c|}{$\mathrm{T}$} & \multicolumn{2}{|c|}{ C } \\
\hline & Domain 1 & Domain 2 & Mean & SD & Mean & $\mathrm{SD}$ & Mean & SD & Mean & SD \\
\hline rTI1B & CTKSNPPTC & CAYSNPPKC & 21 & 2 & 8 & 1 & 2476 & 238 & 2956 & 196 \\
\hline rTI1B mutant & CTGSNPPTC & CAGSNPPKC & \multicolumn{2}{|c|}{ ND } & \multicolumn{2}{|c|}{ ND } & \multicolumn{2}{|c|}{ ND } & \multicolumn{2}{|c|}{ ND } \\
\hline
\end{tabular}

ND, not detected.

* The reactive peptide bond sites are marked in bold text. $\mathrm{K}$ determines specificity for trypsin, whereas $\mathrm{Y}$ determines specificity against chymotrypsin. Amino acid substitutions in the rTI1B mutant are highlighted in grey. 

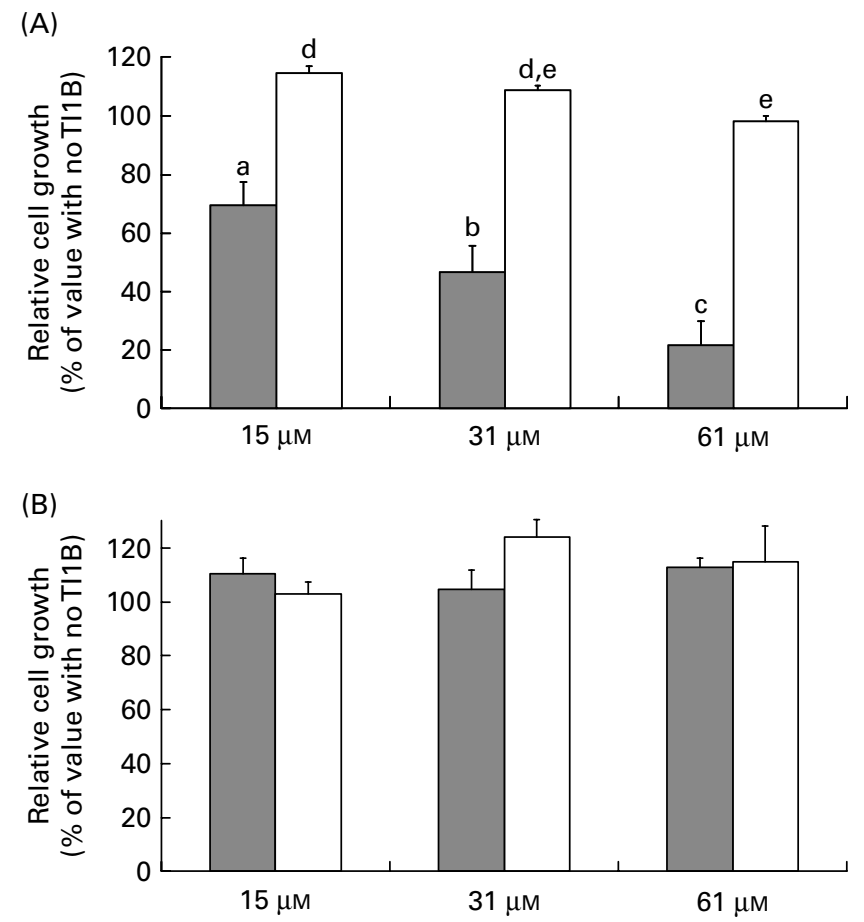

Fig. 4. Effects of recombinant TI1B (rTI1B, closed bars) and the corresponding mutant protein (open bars) on the in vitro growth of (A) HT29 human colorectal adenocarcinoma cells and (B) normal colon fibroblastic CCD-18Co cells. Growth media were supplemented with protein in the concentration range $0-61 \mu \mathrm{M}$ and cells harvested after a period of $96 \mathrm{~h}$. Values are means, with standard deviations represented by vertical bars of at least three independent experiments, each having four technical replicates. ${ }^{a, b, c, d, e}$ Mean values with different letters were significantly different $(P<0.05$; Bonferroni's test).

\section{Effect of $r T / 1 B$ and the corresponding inactive mutant on the proliferation of human colon cells}

The effects of the recombinant proteins on the growth of human colon adenocarcinoma HT29 cells were determined by comparing the cell viability of cultured cells in the absence or presence of rTI1B or the corresponding mutant protein $(0-61 \mu \mathrm{M})$, monitored by the cytotoxic NR cell assay. At concentrations greater than $15 \mu \mathrm{M}$, rTI1B inhibited the in vitro growth of HT29 cells in a concentration-dependent manner (Fig. 4(A)); the $\mathrm{IC}_{50}$ value for rTI1B was 31 (SD 7) $\mu \mathrm{M}$, in agreement with those obtained for BBI from plant sources, including lentil ${ }^{(17)}$ and soyabean ${ }^{(15)}$. In contrast, the growth of HT29 cells was not reduced when treated with the rTI1B related mutant, even at the highest concentration tested $(61 \mu \mathrm{M})$. In addition, the growth of non-malignant CCD-18Co colon cells was unaffected by either rTI1B or its inactive variant in the range of concentrations tested (0-61 $\mu \mathrm{m}$, Fig. 4(B)).

\section{Discussion}

Among other dietary constituents, soyabean BBI and homologous proteins appear to be promising chemopreventive agents within the gastrointestinal tract ${ }^{(5)}$. These dietary constituents, present at high concentrations in legume seeds, have been shown to be effective at preventing or suppressing radiation- and chemical carcinogen-induced transformation, in a wide variety of in vitro assays, and carcinogenesis in in vivo model systems, as reviewed elsewhere ${ }^{(6-8,29,30)}$. In previous studies, we have demonstrated a significant concentrationand time-dependent decrease in the growth of an array of colon cancer cells (HT29, LoVo, Caco2) when treated with BBI proteins from different sources, including soyabean ${ }^{(15)}$, lentil ${ }^{(17)}$ and pea $^{(16)}$; after soyabean BBI treatment, HT29 colon cancer cells showed a dose-dependent increase in the proportion of cells that were arrested in the G0-G1 phase $^{(15)}$. It was shown furthermore that treatment of soyabean BBI with reducing and alkylating agents, which substantially reduces inhibitory activity against serine proteases, renders these dietary proteins unable to inhibit cell proliferation of colon cancer cells ${ }^{(15)}$. Treatments like autoclaving ${ }^{(5)}$ or chemical modification via alkylation of sulphydryl groups ${ }^{(15)}$ have been used as tools for probing the association between protease inhibition and anti-cancer properties of BBI. Related studies have indicated the importance of disulphide bonds in maintaining the three-dimensional structure and inhibitory activity of $\mathrm{BBI}^{(31)}$. Where severe disruptive treatments are used, the native conformation of BBI is lost, alongside losses of tryptic and chymotryptic inhibitory activities, making the relationship between protease inhibition, protein structure and health-beneficial effects unclear. In the present study, we have provided new evidence to support a relationship between the anti-proliferative properties of BBI and their specific inhibition of serine proteases. Here, a loss of native conformation and/or correct protein folding is avoided by comparing the anti-proliferative cellular properties of a major pea isoinhibitor, TI1B, capable of inhibiting both trypsin- and chymotrypsin-like proteases, with a related mutant predicted to lack both TIA and CIA. Both proteins were expressed in recombinant form by heterologous expression in the methylotrophic P. pastoris system, capable of efficiently folding extensively disulphide-bonded proteins, as reported for lentil $\mathrm{BBI}^{(17)}$ and other unrelated protease inhibitors ${ }^{(32)}$. In this study, the masses of the secreted recombinant proteins were consistent with correct disulphide bond formation. When treated with rTI1B, a significant concentration-dependent decrease in the growth of HT29 human colon adenocarcinoma cells was observed, whereas non-malignant colonic fibroblast CCD-18Co cells were unaffected; in contrast, the rTI1B mutant lacking TIA and CIA did not exert any significant effect on the growth of HT29 cells (Fig. 4). These findings suggest that serine proteases should be considered as molecular targets in investigating the potential chemopreventive role of BBI and related proteins during the early stages of carcinogenesis.

Yavelow et $a l{ }^{(33)}$ reported that an enzymatically modified BBI having only CIA was still fully effective as an inhibitor of radiation-induced transformation in vitro, whereas the modified inhibitor with TIA only was ineffective. Although the modified BBI may have been impaired in the inhibition of several molecular targets compared with the native protein, these early observations led to the hypothesis that chymotrypsin-like proteases are potential targets of BBI in anti-cancer effects. In contrast, it has been demonstrated recently that a major soyabean $\mathrm{BBI}$ isoinhibitor which inhibits 
trypsin-like proteases only exerts anti-proliferative properties against colon cancer cells ${ }^{(15)}$. These data suggest that both trypsin- and chymotrypsin-like proteases involved in the early stages of carcinogenesis should be considered as potential targets of BBI-like proteins. So far, the therapeutic targets and the action mechanisms of BBI remain unknown. However, the present study shows that the successful expression of individual isoinhibitors, both naturally occurring and engineered BBI, can assist in understanding the mechanism by which these dietary proteins can inhibit cancer cell proliferation, and in identifying and validating their precise therapeutic targets ${ }^{(6,8)}$. In parallel, direct comparative serine protease profiling studies of malignant and non-malignant cells will explain the differential effects on cell proliferation observed for the variant rTI1B tested here, contributing to the assessment of $\mathrm{BBI}$ for preventive and/or therapeutic medicine.

\section{Acknowledgements}

A. C. acknowledges support by ERDF-co-financed grants from the Spanish CICYT (AGL 2007-60007 and AGL2010-15877) and from CSIC (PIE-200970I054). C. D. acknowledges support from the European Union (Grain Legumes Integrated Project, a Framework Programme 6 project, grant no. FOOD-CT-2004506223) and from Defra (grants nos AR0105 and AR0711). The authors are very grateful to Dr S. Ogueta and A. Lario from the Proteomics Facility of University of Cordoba (Spain) and the Institute of Parasitology and Biomedicine Lopez Neyra (Granada, Spain), respectively, for carrying out peptide mass fingerprinting and molecular mass determination. This study is not subject to any conflicts of interest. A. C. and C. D. were responsible for the experimental design, data analysis, interpretation and writing of the manuscript. M. C. M.-M. was responsible for gene cloning, site-directed mutagenesis and heterologous expression of recombinant proteins. E. J. and M. C. A. carried out protein purification and enzymatic kinetic experiments.

\section{References}

1. Jemal A, Siegel R, Ward E, et al. (2009) Cancer statistics, 2009. Cancer J Clin 59, 225-249.

2. Cummings JH \& Bingham SA (1998) Fortnightly review diet and the prevention of cancer. Br Med J 317, 1636-1640.

3. Reddy BS (2000) Novel approaches to the prevention of colon cancer by nutritional manipulation and chemoprevention. Cancer Epidemiol Biomark Prev 9, 239-247.

4. MacFarlane AJ \& Stover PJ (2007) Factors in gastrointestinal cancers: convergence of genetic, nutritional and inflammatory. Nutr Rev 65, S157-S166.

5. Kennedy AR, Billings PC, Wan XS, et al. (2002) Effects of Bowman-Birk inhibitor on rat colon carcinogenesis. Nutr Cancer 43, 174-186.

6. Clemente A \& Domoney C (2006) Biological significance of polymorphism in plant protease inhibitors from the Bowman-Birk class. Curr Prot Pept Sci 7, 201-216.

7. Clemente A \& Domoney C (2007) Therapeutic properties of legume protease inhibitors from the Bowman-Birk class. In Recent Progress in Medicinal Plants, pp. 397-417 [JN Govil,
VK Singh and RK Sharma, editors]. vol. 20, Houston, TX: Studium Press.

8. Clemente A, Sonnante G \& Domoney C (2011) BowmanBirk inhibitors from legumes and human gastrointestinal health: current status and perspectives. Curr Prot Pept Sci 12, 358-373.

9. Chen P, Rose J, Love R, et al. (1992) Reactive sites of an anticarcinogenic Bowman-Birk proteinase inhibitor are similar to other trypsin inhibitors. J Biol Chem 267, 1990-1994.

10. Ramasarma PR, Appu Rao AG \& Rao DR (1995) Role of disulfide linkages in structure and activity of proteinase inhibitor from horsegram (Dolichos biflorus). Biochim Biophys Acta 1248, 35-42.

11. Trivedi MV, Laurence JS \& Siahann TJ (2009) The role of thiols and disulfides on protein stability. Curr Prot Pept Sci 10, 614-625.

12. Clemente A, Jimenez E, Marín-Manzano MC, et al. (2008) Active Bowman-Birk inhibitors survive gastrointestinal digestion at the terminal ileum of pigs fed chickpea-based diets. J Sci Food Agric 88, 523-531.

13. Marín-Manzano MC, Ruiz R, Jimenez E, et al. (2009) Anticarcinogenic soyabean Bowman-Birk inhibitors survive faecal fermentation in their active form and do not affect the microbiota composition in vitro. Br J Nutr 101, 967-971.

14. Kennedy AR, Szuhaj BF, Newberne PM, et al. (1993) Preparation and production of a cancer chemopreventive agent, Bowman-Birk inhibitor concentrate. Nutr Cancer 19, 281-302.

15. Clemente A, Moreno J, Marín-Manzano MC, et al. (2010) The cytotoxic effect of Bowman-Birk isoinhibitors from soybean on HT29 human colorectal cancer cells is related to their intrinsic ability to inhibit serine proteases. Mol Nutr Food Res 54, 396-405.

16. Clemente A, Gee JM, Johnson IT, et al. (2005) Pea (Pisum sativum L.) protease inhibitors from the Bowman-Birk class influence the growth of human colorectal adenocarcinoma HT29 cells in vitro. J Agric Food Chem 53, 8979-8986.

17. Caccialupi P, Ceci LR, Siciliano RA, et al. (2010) BowmanBirk inhibitors in lentil: heterologous expression, functional characterisation and anti-proliferative properties in human colon cancer cells. Food Chem 120, 1058-1066.

18. Domoney C. (1999) Inhibitors of legume seeds. In Seed Proteins, pp. 635-655 [PR Shewry and R Casey, editors]. The Netherlands: Kluwer Academic Publishers.

19. Domoney C, Welham T, Ellis N, et al. (2002) Three classes of proteinase inhibitor gene have distinct but overlapping patterns of expression in Pisum sativum plants. Plant Mol Biol 48, 319-329.

20. Ho S, Hunt H, Horton R, et al. (1989) Site-directed mutagenesis by overlap extension using the polymerase chain reaction. Gene $77,51-59$.

21. Domoney C \& Welham T (1992) Trypsin inhibitors in Pisum: variation in amount and pattern of accumulation in developing seed. Seed Sci Res 2, 147-154.

22. Clemente A, MacKenzie DA, Jeenes DJ, et al. (2004) The effect of variation within inhibitory domains on the activity of pea protease inhibitors from the Bowman-Birk class. Protein Express Purif 36, 106-114.

23. Copeland RA, Lombardo D, Giannaras J, et al. (1995) Estimating $K_{\mathrm{i}}$ values for tight-binding inhibitors from doseresponse plots. Bioorg Med Chem Lett 5, 1947-1952.

24. Domoney C, Welham T, Sidebottom C, et al. (1995) Multiple isoforms of Pisum trypsin inhibitors result from modification of two primary gene products. FEBS Lett 360, 15-20. 
25. Schechter I \& Berger A (1967) On the size of the active site in proteases. I. Papain. Biochem Biophys Res Commun 27, 157-162.

26. Damaso MC, Almeida MS, Kurtenbach E, et al. (2003) Optimized expression of a thermostable xylanase from Thermomyces lanuginosus in Pichia pastoris. Appl Environ Microbiol 69, 6064-6072.

27. Chen Z, Wang D, Cong Y, et al. (2011) Recombinant antimicrobial peptide hPAB- $\beta$ expressed in Pichia pastoris, a potential agent active against methicillin-resistant Staphylococcus aureus. Appl Microbiol Biotechnol 89, 281-291.

28. Scarafoni A, Consonni A, Galbusera V, et al. (2008) Identification and characterization of a Bowman-Birk inhibitor active towards trypsin but not chymotrypsin in Lupinus albus seeds. Phytochem 69, 1820-1825.

29. Kennedy AR (1998) Chemopreventive agents: protease inhibitors. Pharmacol Ther 78, 167-209.
30. Kennedy AR (1998) The Bowman-Birk inhibitor from soybeans as an anticarcinogenic agent. Am J Clin Nutr $\mathbf{6 8}$, 1406s-1412s.

31. Singh RR \& Appu Rao AG (2002) Reductive unfolding and oxidative refolding of a Bowman-Birk inhibitor from horsegram seeds (Dolichos biflorus): evidence for "hyperreactive" disulfide bonds and rate-limiting nature of disulfide isomerisation in folding. Biochim Biophys Acta 1597, 280-291.

32. Volpicella M, Ceci LR, Cordewener J, et al. (2003) Properties of purified gut trypsin from Helicoverpa zea, adapted to protease inhibitors. Eur J Biochem 270, 10-19.

33. Yavelow J, Collins M, Birk Y, et al. (1985) Nanomolar concentrations of Bowman-Birk soybean protease inhibitor suppress X-ray induced transformation in vitro. Proc Natl Acad Sci U S A 82, 5395-5399. 